\title{
Fixation par ancrages d'une canalisation de gaz enterrée dans un terrain inondable
}

P. HABIB

LMS-G3S

École polytechnique 91128 Palaiseau Cedex

S. AKEL

B. DESTOMBES

M. ZAREA

GDF, Direction

de la Recherche

361, av. du Président-

Wilson

93211 La Plaine-Saint-Denis

Cedex

\section{J. BICHET}

G. CHATELET

GDF, DPT

Centre National

d'Équipement

5, rue Pierre-Bérégovoy

92111 Clichy

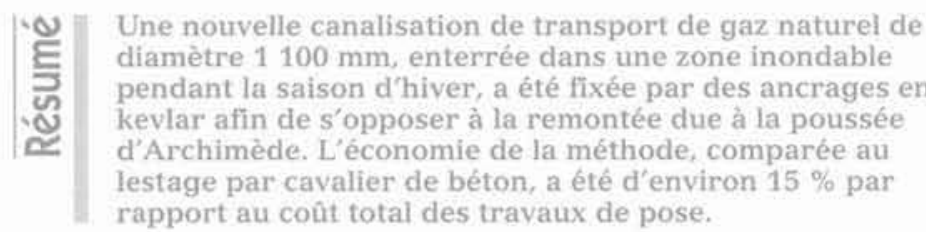

rapport au coût total des travaux de pose.

\section{A gas pipeline buried in flooded soil held with anchorage}

A large diameter new gas pipeline buried in a flooded area during the winter season has been held with kevlar anchorage to prevent uplift pipe movement due to buoyancy. This method is much less expensive than placing additional weight on the pipeline. The saving was about $15 \%$ of the total cost of the laying of the pipeline. 


\section{Introduction}

"L'Artère des Hauts de France » est une canalisation de gaz enterrée à un mètre de profondeur, destinée à acheminer le gaz norvégien de la mer du Nord à partir d'un terminal situé près de Dunkerque vers le réseau français. Le gaz naturel sera ainsi transporté sur $185 \mathrm{~km}$ dans une conduite de $1100 \mathrm{~mm}$ de diamètre sous une pression de $8,5 \mathrm{MPa}$.

Au départ de Dunkerque et sur une distance de $13 \mathrm{~km}$ environ, cette canalisation traverse un site très plat constitué de sols plus ou moins limoneux ou sableux, où la nappe phréatique fluctue suivant les saisons et les pluies jusqu'à atteindre le niveau du sol : en fait, cette zone est pratiquement inondable en hiver. La canalisation est ainsi soumise d'une façon cyclique à la poussée d'Archimède. Dans cette zone, le volume extérieur de la canalisation (y compris le revêtement de protection) varie entre $1,008 \mathrm{~m}^{3}$ par mètre linéaire pour les tubes de catégorie d'emplacement B et 1,039 $\mathrm{m}^{3}$ par mètre linéaire pour les tubes de catégorie d'emplacement $C$ pour des poids linéiques moyens de $4925 \mathrm{~N} / \mathrm{ml}$ et de $7123 \mathrm{~N} / \mathrm{ml}$ respectivement (la catégorie d'emplacement se traduit par des épaisseurs différentes pour les canalisations), La poussée d'Archimède est donc voisine de $10000 \mathrm{~N} / \mathrm{ml}$ en négligeant le poids du gaz sous pression, ce qui implique un déficit fondrier de l'ordre de $5000 \mathrm{~N} / \mathrm{ml}$ pour les tubes de catégorie $\mathrm{B}$ et $3000 \mathrm{~N} / \mathrm{ml}$ pour les tubes de catégorie C.

\section{2}

\section{Principe de la fixation de la canalisation}

La solution classique pour immobiliser une canalisation enterrée est de la lester; généralement par du béton ; cette solution est sûre mais présente des inconvénients, en particulier si une intervention au niveau de la protection devenait nécessaire.

Il a paru plus économique d'envisager un procédé original au moyen d'ancrages précontraints dont le principe est indiqué ci-dessous. De part et d'autre de la canalisation, on installe des points d'ancrage (Fig. 1) constitués par des ancres métalliques articulées, mises en place par battage, qui basculent et se coincent dans le sol lorsqu'on les tire en arrière par la ligne d'accrochage, après avoir été battues à la profondeur désirée (Fig. 2).

Ces couples d'ancres sont reliés par une sangle en kevlar passant au-dessus de la canalisation (Fig. 3) que l'on peut mettre en tension par un dispositif de serrage manuel. La distance entre les couples d'ancres est liée à la résistance qu'on peut espérer en sécurité pour les ancrages (Fig. 4).

L'emploi de sangles en kevlar a été retenu de façon à éviter la corrosion dans le sol de la ligne d'ancrage d'une part et, d'autre part, pour ne pas avoir un contact trop rude avec le revêtement protecteur de la canalisation. La conception de la sécurité de l'ensemble du dispositif est inspirée des « Recommandations du CFMS pour les tirants d'ancrage - TA 95 » en restant systématiquement prudent pour les coefficients de sécurité du fait que la sollicitation par la poussée d'Archimède n'est pas statique mais répétée. Ce règlement et le mode de mise en place imposent un certain nombre de précautions :

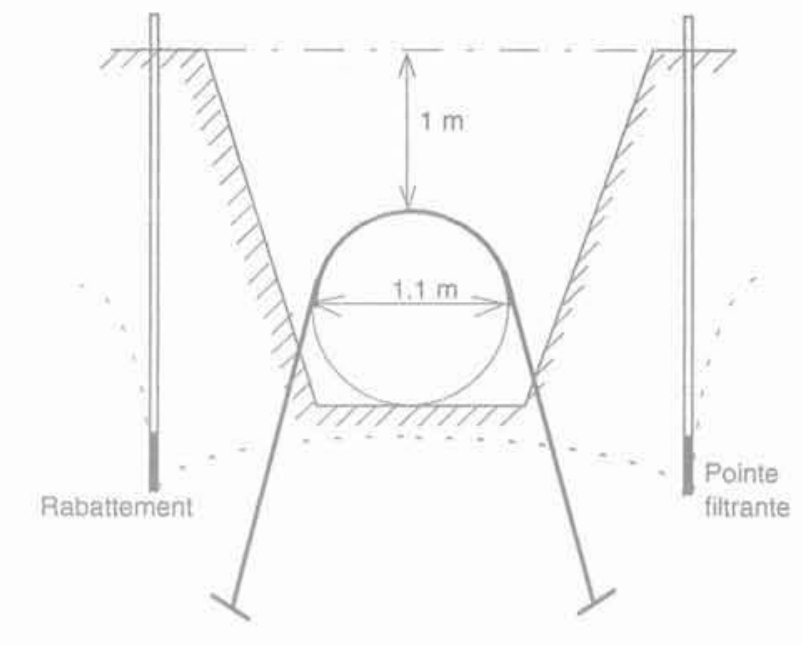

FIG, 1 Principe de fixation par ancrages. Pipeline held with anchorages.
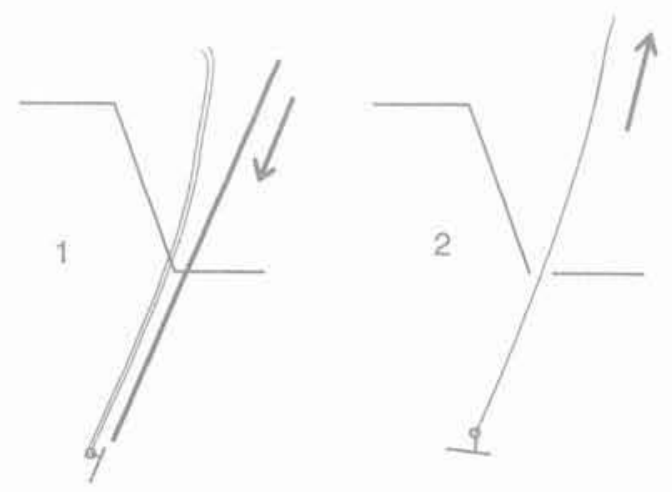

FIG, 2 Mise en place des ancres (1) battage (2) basculement. Putting of the anchorage in its place (1) driving (2) rocking.

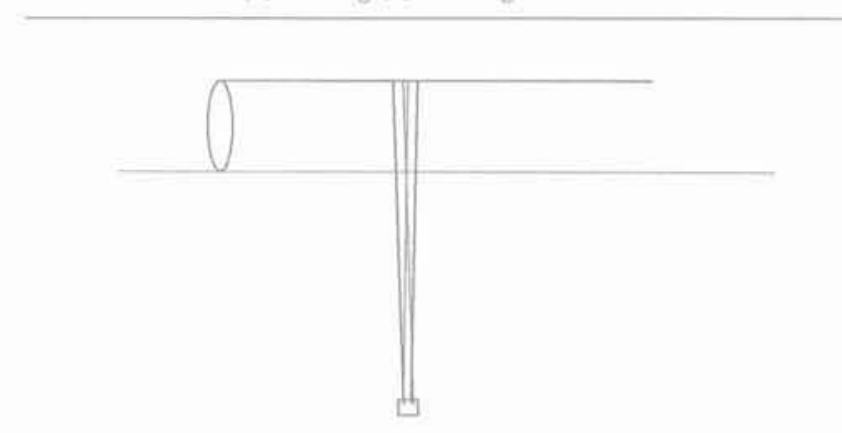

FIG. 3 Mise en place de la sangle. Putting of the kevlar strap over the pipeline.

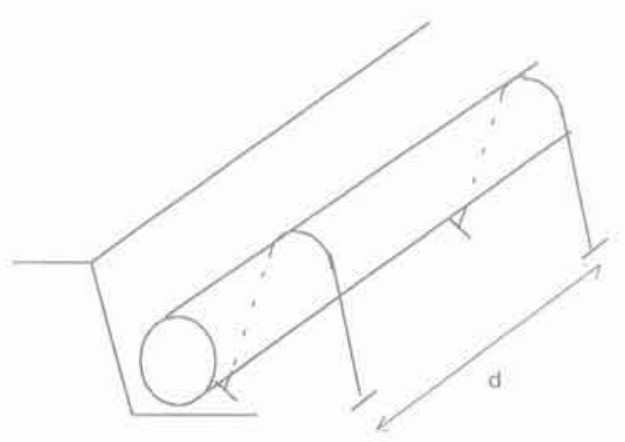

FIG. 4

Distance d entre les couples d'ancres. Distance d between couples of anchors. 
- d'abord des essais préalables systématiques d'arrachage des ancres chaque fois que le tracé rencontre un nouveau terrain, selon les procédures TA95 et de la norme NFP 94-153 pour définir la traction de service;

- ensuite, à partir de l'énergie de battage pour enfoncer les ancres, il faut évaluer la force d'enfoncement de façon à relier empiriquement la force d'enfoncement à la force ultime d'arrachage des ancres, ceci étant occasionnellement confirmé par des essais au pénétromètre dynamique ;

- ensuite encore, une épreuve de mise en charge de chaque ancre au moment du basculement des ancres, avant la mise en tension des ancres :

- enfin, un contrôle de la tension des sangles en fonction du temps et du niveau de la nappe sur un nombre limité de couples d'ancres, répartis le long du tracé de la canalisation au moyen de dynamomètres à corde vibrante.

\section{3}

\section{Les essais préliminaires}

\section{1}

\section{Essais d'arrachage}

Le sol, sous le niveau inférieur de la canalisation, est constitué d'un horizon de sable gris permettant un bon accrochage des ancres. On a tout d'abord vérifié avec différents modèles d'ancres qu'il était possible dans ce terrain, à deux mètres de profondeur, d'obtenir une résistance de l'ordre de $60 \mathrm{kN}$ avec une procédure conforme au règlement sur les tirants d'ancrage. D'une façon générale, les ancrages au sol sont plutôt surabondants. En effet, les sangles sont en kevlar préétiré pour éliminer le caractère non linéaire du premier chargement, et on a retenu un coefficient de sécurité de lordre de 10 pour éviter tout risque de fluage ultérieur ou de fatigue, alors qu'on se contente d'un coefficient de sécurité de 2 pour lancrage : ce coefficient de 2 est choisi de façon qu'en cas de défaillance complète d'un ancrage, pour une cause accidentelle, la force manquante se répartissant entre les encadrants, il reste alors sur les ancrages voisins un coefficient de sécurité de 1,33 par rapport à la traction de service, ce qui est bien suffisant. De sorte que, si au cours des essais préalables, on n'arrive pas à arracher l'ancre, en étant limité par la résistance du kevlar, on est sûr que la qualité du sol est très satisfaisante.

Les essais préalables ont comporté aussi des essais de longue durée qui ont montré qu'il n'y avait pas de perte de tension en fonction du temps par fluage du sol au voisinage de l'ancre, ce qui est tout à fait normal pour un sol sableux.

\section{2}

\section{Essai en vraie grandeur}

Un essai en vraie grandeur de type industriel a été effectué avant le lancement des travaux de façon à confirmer la faisabilité de la mise en place. Pour cela un tronçon de canalisation de $50 \mathrm{~m}$ de long (avec deux fonds) a été installé dans une tranchée de $1,90 \mathrm{~m}$ de profondeur, analogue à celle du chantier de pose ; 7 couples d'ancres ont été battus avec un espacement de $7 \mathrm{~m}$, et les sangles ont êté mises en place au-dessus de la canali- sation, puis la tranchée a été inondée soumettant ainsi la canalisation à la pleine poussée d'Archimède. La canalisation était instrumentée par des extensomètres à résistance électrique de façon à mesurer les déformations dans l'acier au voisinage des sangles. Enfin, la sangle du couple d'ancres central a été coupée pour voir le report sur les sangles voisines et l'effet sur les contraintes dans l'acier de la canalisation. Ces essais ont montré que le surcroit de contrainte dans l'acier est peu important; en définitive, c'est surtout la qualité du sol et la résistance des ancrages qui imposent la distance entre les sangles. A la suite de ces essais, la distance entre les couples d'ancrages a été fixée à $8,50 \mathrm{~m}$ pour l'ouvrage définitif.

\section{4}

\section{La réalisation}

La pose de la canalisation et des ancrages a été faite par l'entreprise Denys dans une tranchée protégée par un rabattement de nappe local (Fig. 1).

Une reconnaissance géotechnique préalable du tracé avait été faite, notamment au moyen d'essais de pénétration. Mais, quelle que soit la qualité de la reconnaissance et quelle que soit la régularité de la nature géologique du terrain, on ne peut pas supposer, avant l'exécution du travail, que le sol soit rigoureusement le même sur tout le tracé, et que des points d'ancrages situés tous les $8,50 \mathrm{~m}$ soient exactement décrits par la reconnaissance préalable. D'où la nécessité de tester chaque ancrage au moment de la mise en place des ancres lorsqu'on tire les ancres en arrière pour les faire basculer, en plus de l'estimation de la force d'enfoncement par la mesure de l'énergie de battage. Mais que faudrait-il faire si le sol au voisinage d'un emplacement d'ancrage s'avérait défectueux? 'Trois façons d'y remédier sont possibles :

1 - Enfoncer les ancres plus profondément pour rechercher le bon sol: pour cela, il faut disposer d'un faux-pieu plus long ou le rabouter et couper à la demande des sangles en kevlar plus longues.

2 - Diminuer la distance entre les couples d'ancrages, ce qui revient à mettre davantage d'ancres. Il parait peu souhaitable de réduire leur distance à moins de $4 \mathrm{~m}$.

3 -Disposer sur le chantier d'ancres de plus grande surface. En effet, il y a probablement peu de danger à craindre avec des sols sableux, et c'est davantage une argile un peu molle qui peut présenter une résistance insuffisante. Or, dans ce cas, la résistance est proportionnelle à la surface de l'ancre.

Chacun de ces remèdes peut permettre de faire mieux que doubler la résistance des ancrages et rien n'empêche de les utiliser simultanément. De sorte que la sécurité opérationnelle du système de fixation peut être facilement multipliée par 10, si le besoin s'en faisait sentir. C'est évidemment largement suffisant pour faire face aux pires situations, étant par ailleurs bien entendu qu'en aucun cas les ancres ne doivent être placées dans de la tourbe, dont les caractéristiques mécaniques à long terme sont toujours désastreuses.

\section{5}

\section{Précontrainte des ancrages}

La précontrainte du système ancres-sangle est indispensable pour une immobilisation pratique de la canalisation par rapport au sol. En effet, lorsque le 


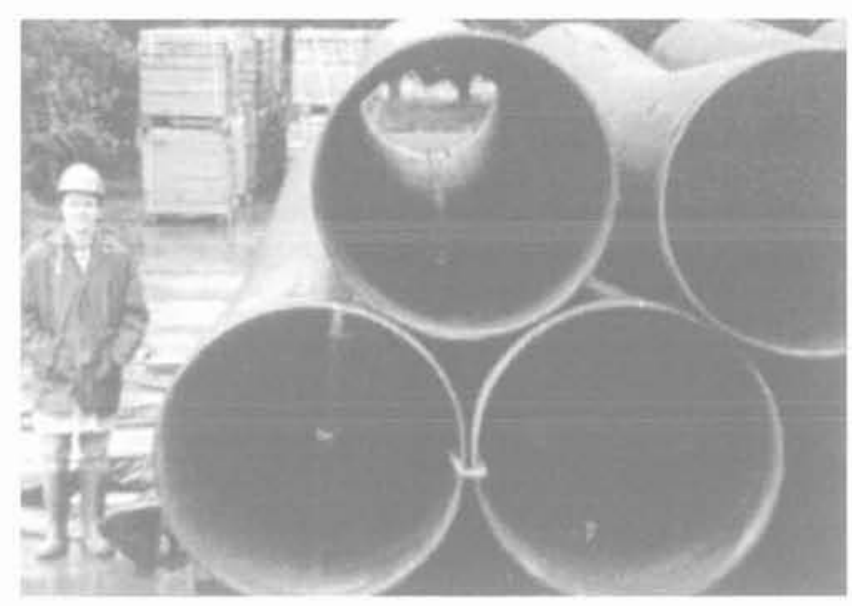

PHOTO1 Tubes cintrés sur parc.

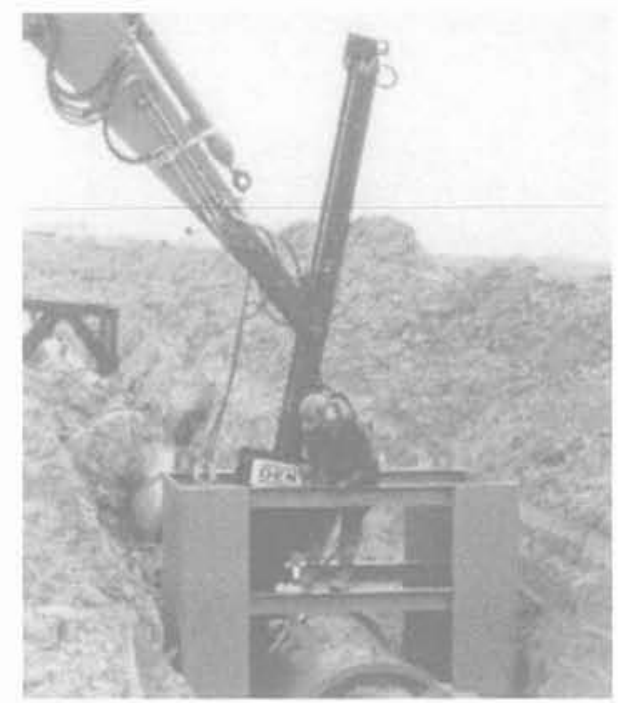

PHOTO 3 Battage des ancres, la conduite étant protégée par un châssis.

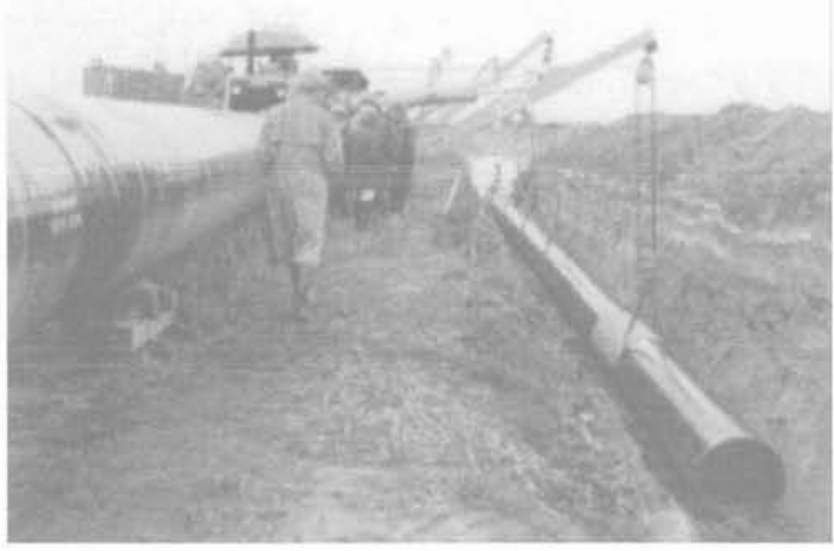

PHoro2 Mise en place de la conduite.

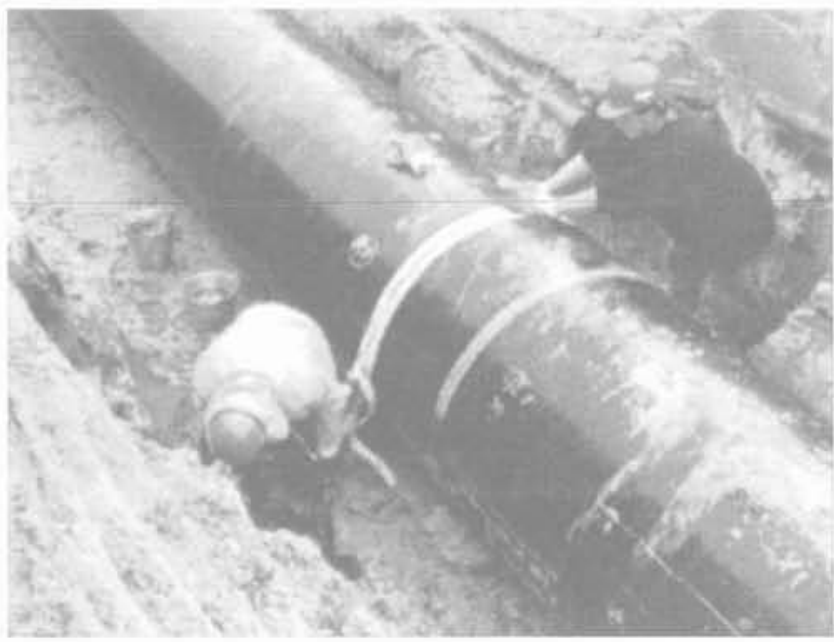

PHOTO 4 Mise en place des ancres de kevlar.

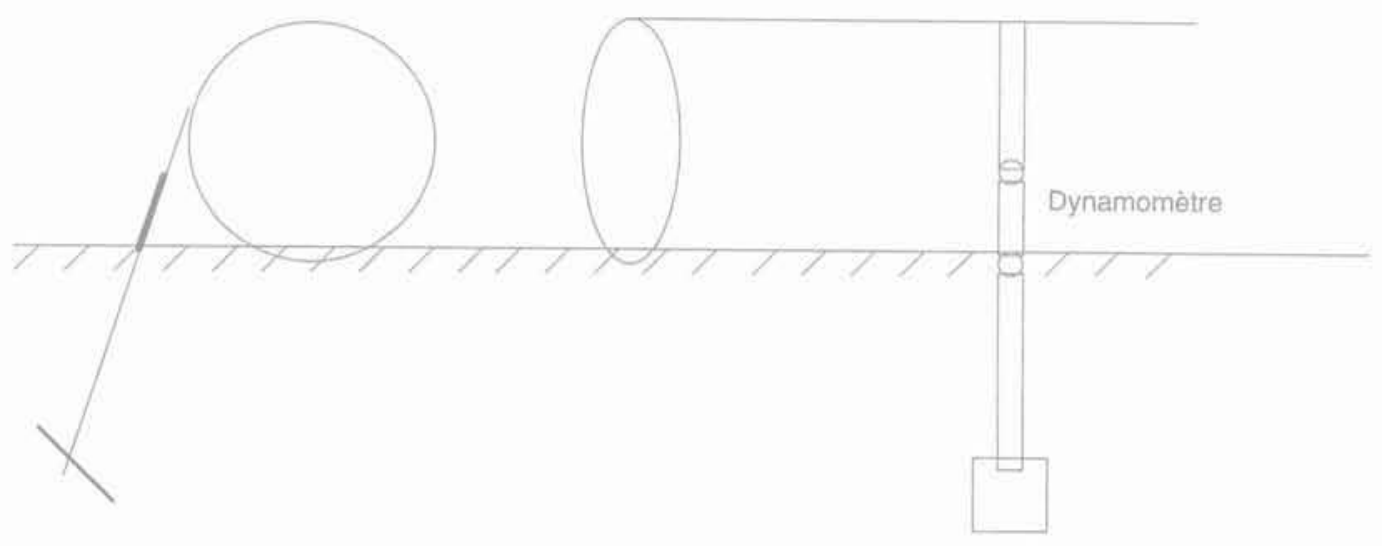

FIG.5 Dynamomètre à corde vibrante installé sur une sangle.

Vibrating wire dynamometer put in place in a strap.

niveau de la nappe monte, la canalisation est soumise progressivement à une force ascendante égale à la différence entre la poussée d'Archimède et le poids de la deux ancres sont alors sollicitées par $5000 \mathrm{~N} / \mathrm{m}$ $\times 8,5 \mathrm{~m}=42500 \mathrm{~N}$, soit $21250 \mathrm{~N}$ par ancre et, par conséquent, chaque sangle est tendue à $10625 \mathrm{~N}$. Avec un coefficient d'élasticité de $2,7.10^{-7} \mathrm{~N}$ et une demi-lon- 


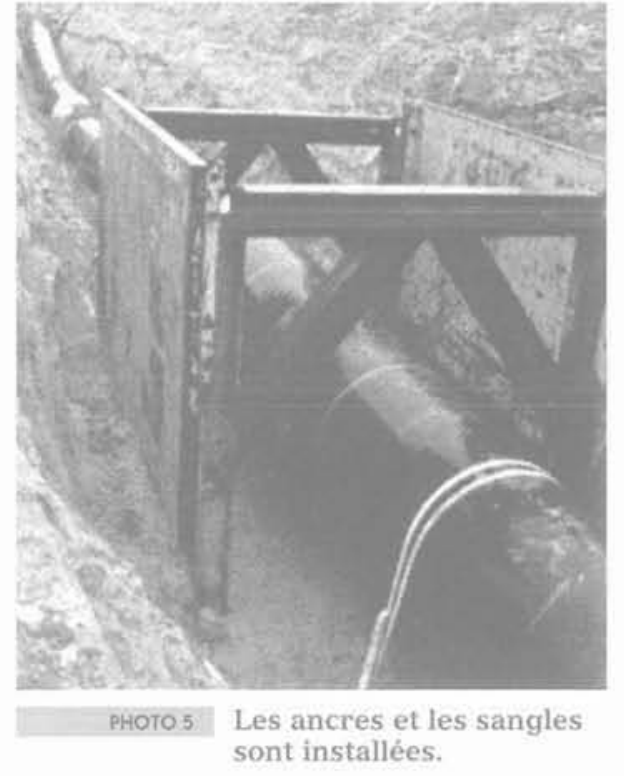

gueur de sangle d'environ $4 \mathrm{~m}$, le déplacement de la canalisation provoqué par l'allongement de la sangle serait de l'ordre de $1 \mathrm{~cm}$ ce qui n'est pas satisfaisant pour une sollicitation cyclique. Au contraire, si les sangles sont tendues par une précontrainte égale ou supérieure à $10625 \mathrm{~N}$, la canalisation est parfaitement fixée au déplacement près de la canalisation sur son appui sur le sol.

Le calcul montre d'ailleurs que ce déplacement est inférieur au millimètre, c'est-à-dire parfaitement négligeable, du fait que le module d'élasticité du sol sous l'effet d'un chargement cyclique est beaucoup plus grand que le module de premier chargement.

Ainsi pour éviter des mouvements notables de la canalisation, il est indispensable que sa fixation soit précontrainte et que cette force se maintienne sans que des phénomènes de fluage se produisent, c'est d'ailleurs la raison pour laquelle des coefficients de sécurité élevés ont été choisis. Il est cependant nécessaire de s'assurer de la constance de cette tension en fonction du temps. Pour cela, en suivant les recommandations du règlement sur les ancrages, une dizaine de couples d'ancres équipés de dynamomètre à cordes

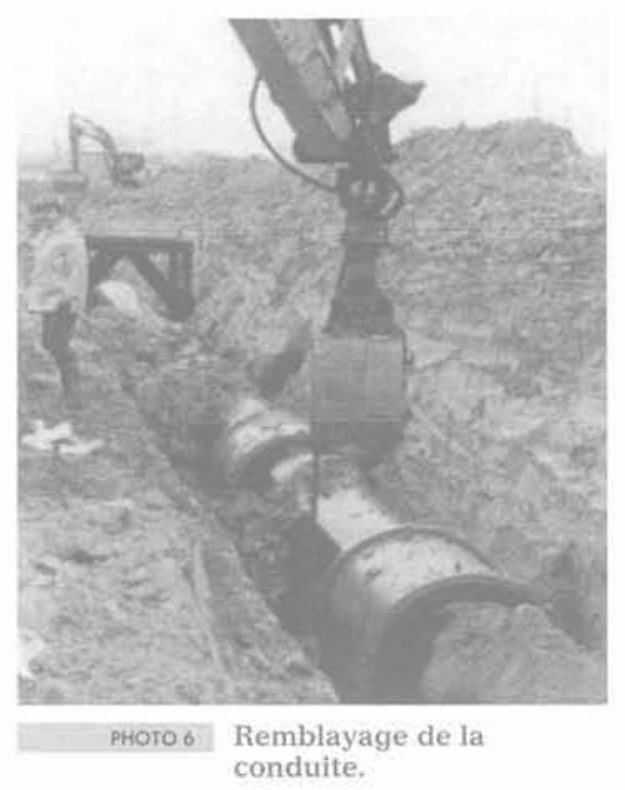

vibrantes a été mise en place pour suivre l'évolution de la tension en fonction de la montée du plan d'eau (Fig. 5),

La mise en place de la canalisation et des ancrages a été faite au cours de l'été et de l'automne 1997. Actuellement, en 1998, un cycle saisonnier est passé. Les mesures faites par la topographie et celles faites avec les dynamomètres n'ont rien montré qui soit inquiétant. Le suivi des observations se poursuit. Les photographies suivantes illustrent le déroulement du chantier.

L'utilisation de 1650 couples d'ancres a nécessité près de 20000 mètres de sangle en kevlar et a permis d'éviter la mise en place d'un lestage de $1000 \mathrm{~kg} \cdot \mathrm{m}^{-1}$ environ (en tenant compte des coefficients de sécurité imposés par Gaz de France) sur $13 \mathrm{~km}$, soit $16,106 \mathrm{~kg}$ représentant environ $6500 \mathrm{~m}^{3}$ de béton.

Outre une réduction importante des impacts sur l'environnement (pas de surprofondeur, limitation des pompages, conservation de l'intégrité de la piste de roulement, etc.), l'utilisation de cette technique de préférence à une solution traditionnelle a permis de réaliser un gain financier de l'ordre de $8 \mathrm{MF}$, soit environ $15 \%$ du cout des travaux de pose dans cette zone. 